\title{
An adaptive least angle regression method for uncertainty quantification in FDTD computation
}

DOI:

10.1109/TAP.2018.2872161

\section{Document Version}

Accepted author manuscript

Link to publication record in Manchester Research Explorer

\section{Citation for published version (APA):}

Hu, R., Costen, F., Monebhurrun, V., Himeno, R., \& Yokota, H. (2018). An adaptive least angle regression method for uncertainty quantification in FDTD computation. IEEE Transactions on Antennas and Propagation .

https://doi.org/10.1109/TAP.2018.2872161

\section{Published in:}

IEEE Transactions on Antennas and Propagation

\section{Citing this paper}

Please note that where the full-text provided on Manchester Research Explorer is the Author Accepted Manuscript or Proof version this may differ from the final Published version. If citing, it is advised that you check and use the publisher's definitive version.

\section{General rights}

Copyright and moral rights for the publications made accessible in the Research Explorer are retained by the authors and/or other copyright owners and it is a condition of accessing publications that users recognise and abide by the legal requirements associated with these rights.

\section{Takedown policy}

If you believe that this document breaches copyright please refer to the University of Manchester's Takedown Procedures [http://man.ac.uk/04Y6Bo] or contact uml.scholarlycommunications@manchester.ac.uk providing relevant details, so we can investigate your claim.

\section{OPEN ACCESS}




\title{
An adaptive least angle regression method for uncertainty quantification in FDTD computation
}

\author{
Runze Hu, Fumie Costen, Senior Member, IEEE, Vikass Monebhurrun, Senior Member, IEEE, Ryutaro Himeno, \\ Hideo Yokota
}

\begin{abstract}
The non-intrusive polynomial chaos (NIPC) expansion method is used to quantify the uncertainty of a stochastic system. It potentially reduces the number of numerical simulations in modelling process, thus improving efficiency, whilst ensuring accuracy. However, the number of polynomial bases grows substantially with the increase of random parameters, which may render the technique ineffective due to the excessive computational resources. To address such problems, methods based on the sparse strategy such as the least angle regression (LARS) method with hyperbolic index sets can be used. This paper presents the first work to improve the accuracy of the original LARS method for uncertainty quantification (UQ). We propose an adaptive LARS method in order to quantify the uncertainty of the results from the numerical simulations with higher accuracy than the original LARS method. The proposed method outperforms the original LARS method in terms of accuracy and stability. The $L 2$ regularisation scheme further reduces the number of input samples while maintaining the accuracy of the LARS method.
\end{abstract}

Index Terms-Non-intrusive polynomial chaos (NIPC) expansion, least angle regression (LARS), uncertainty quantification (UQ), finite difference time domain (FDTD), Debye media

\section{INTRODUCTION}

Benefiting from the rapid development of communication technology, wireless communication devices such as mobile phones [1], [2] have grown in popularity, raising concern about the potential dangers of exposure to electromagnetic radiation [3], [4]. Tissues in the immediate vicinity absorb the radio frequency (RF) energy emitted from the wireless devices, which may potentially be hazardous [5]. To evaluate the energy absorption when the human body is exposed to an $\mathrm{RF}$ electromagnetic field, the specific absorption rate (SAR) [6], [7] is measured using a standard dosimetric test facility. However, since the SAR test is expensive and time-consuming, the use of electromagnetic simulation to model the RF energy exposure is a preferred alternative.

The finite difference time domain (FDTD) [8], [9] method has a powerful and robust performance in modeling inhomo-

R. Hu and F. Costen are with the School of Electrical and Electronic Engineering, The University of Manchester, U.K. (email: fumie.costen@manchester.ac.uk).

V. Monebhurrun is with the EXPOSE/PIEM/GEEPS, CentraleSupelec, 11 rue Joliot Curie Plateau de Moulon, Gif sur Yvette 91192, France (e-mail: Vikass.MONEBHURRUN@ centralesupelec.fr).

R. Himeno is with Head Office for Information Systems and Cybersecurity, RIKEN, Saitama, Japan.

H. Yokota and F. Costen are with the Image Processing Research Team, Centre for Advanced Photonics, RIKEN, Saitama, Japan.

Color version of the figures in this paper are available online at http://ieeexplore.ieee.org.

Additional research data supporting this publication are available from http://dx.doi.org/ repository at 10.17632/tpvrtz3w6z.1. geneous materials and the simulation of wideband antennae, which makes it a suitable method for bioelectromagnetic simulations. To explore the propagation of the electromagnetic waves in human body, a digital human phantom (DHP) is introduced into the FDTD space. One of the main advantages [10] of utilising a DHP is that it allows researchers to test the electromagnetic response without exposing human body to the electromagnetic radiation.

In the FDTD calculations, the one-pole Debye model [11] is utilised to describe the complex frequency-dependent behaviour of biological tissues. Each tissue in the DHP is associated with four Debye parameters and these parameters differ between people. Such variation is taken into account by introducing uncertainty. In the numerical simulations, the Debye parameters are considered as the input parameters of the system used to determine the electromagnetic response of the human body. The uncertainty of these input parameters can be described by their probabilistic description [12]. However, uncertainty also exists in the system response, due to the propagation of the randomness of the input parameters.

This paper focuses on the uncertainty quantification (UQ) of the FDTD method. The traditional approach for UQ is the Monte Carlo method (MCM) [13], [14], yet this technique requires a large number of simulations resulting in computational inefficiency. Although methods such as the Latin Hypercube sampling (LHS) method [15] and the Markov Chain Monte Carlo (MCMC) method [16] are capable of speeding up the convergence rate of the MCM method, the applications of MCM are very limited because the computational requirements are still excessive.

The non-intrusive polynomial chaos (NIPC) expansion method [17], [18] is one of the most frequently used methods for UQ as an alternative to MCM. We obtain the coefficients of the NIPC method mainly from the Galerkin projection method [19] or the regression method [20]. Nevertheless, both methods are prone to the curse of dimensionality, whereby the number of calculations grows with the increase of the number of input parameters, leading to the rise of the computational cost. A solution to this problem was proposed in [18] wherein each parameter is considered one at a time.

To circumvent the curse of dimensionality, a truncation scheme known as the hyperbolic polynomial chaos expansion [21] is applied in this work. This reduces the number of polynomial bases, based on the sparsity-of-effects principle. When we have multiple variables, the higher-order Hermite polynomials may be less significant than the lower-order ones [22]. Thus, the sparse polynomial chaos (PC) expansion can be built by discarding less important polynomial bases without 
sacrificing accuracy. To quantify the uncertainty of the FDTD results using only significant Hermite polynomial bases, a classical model selection method, known as the least angle regression (LARS) method [21], [23], is undertaken. Given a set of possible polynomial bases, the LARS method aims to select those polynomial bases which possess high correlation with the output of the system. The LARS procedure consists of a number of iterations, each of which is concerned with the selection of the most correlated polynomial bases. A termination criterion is usually set in order to control the LARS procedure, thereby resulting in the selection of only a limited number of polynomial bases. Such features render the LARS method computationally efficient. However, the LARS method has never been applied to quantify the uncertainty of the FDTD results.

This paper proposes an adaptive LARS method for our inhouse FDTD code mainly focusing on the improvement of the accuracy of the LARS method. To successfully perform the matrix inversion in the LARS method, the number of input samples must exceed the number of polynomial bases. Such a condition restricts the minimum number of input samples. However, when the condition is not met, this does not necessarily imply that the LARS method underperforms or is excessively inaccurate. To explore the relationship between the accuracy of the LARS method and the required number of input samples, the L2 regularisation scheme [24] is incorporated into the LARS method to enable the technique to function irrespective of the number of input samples. As a result, the number of input samples may be further reduced without degrading the accuracy of the LARS method. The paper is organised as follows: Section II explains the principle of the hyperbolic scheme, the LARS method and proposes the adaptive LARS method. Section III details the numerical experiments for UQ by MCM, LARS and the adaptive LARS methods. Finally, we analyse the results from these techniques and emphasise the merit of the adaptive LARS method.

\section{Methodology}

The hyperbolic scheme enables us to build a sparse PC expansion. The LARS and adaptive LARS methods are used to quantify the uncertainty of the FDTD results.

\section{A. The non-intrusive polynomial chaos expansion}

The NIPC method approximates the random outputs of the system using a series of orthogonal polynomials. In the NIPC method, quantifying the uncertainty of a system is equivalent to the calculation of the coefficients of polynomial bases [25]. The polynomial basis is defined as $\psi_{\alpha_{1} \alpha_{2} \cdots \alpha_{\mathcal{K}}}\left(\xi_{1}, \xi_{2}, \cdots, \xi_{\mathcal{K}}\right)$, where $\alpha_{1} \alpha_{2} \cdots \alpha_{\mathcal{K}}$ is the index of the polynomial basis, and $\xi_{1}, \xi_{2}, \cdots, \xi_{\mathcal{K}}$ are $\mathcal{K}$ variables. In this paper, each variable $\xi_{k}$ represents a certain Debye parameter of interest of a certain tissue and we observe the electric field $\boldsymbol{E}$ in the FDTD simulations. The highest order of the polynomial basis in a PC expansion is denoted as $r \in[1,+\infty) . r$ is set to a certain finite value so that the PC expansion is truncated from the infinite PC expansion. In this case, $\alpha_{k}$ satisfies

$$
\sum_{k=1}^{\mathcal{K}} \alpha_{k} \leq r, \alpha_{k} \geq 0
$$

and the total number of polynomial bases in the NIPC expansion is

$$
\mathcal{L}=\frac{(\mathcal{K}+r) !}{\mathcal{K} ! r !}
$$

For example, in the case of $r=2$ and $\mathcal{K}=3$, the number of polynomial bases in the PC expansion is $\mathcal{L}=10$ in (2), and the Hermite polynomials $\psi_{\alpha_{1} \alpha_{2} \alpha_{3}}\left(\xi_{1}, \xi_{2}, \xi_{3}\right)$ are shown in

\begin{tabular}{|c|c|}
\hline Hermite polynomials & The order of $\psi$ \\
\hline$\psi_{000}\left(\xi_{1}, \xi_{2}, \xi_{3}\right)=\psi_{0}\left(\xi_{1}\right) \psi_{0}\left(\xi_{2}\right) \psi_{0}\left(\xi_{3}\right)=1$ & 0 \\
\hline$\psi_{100}\left(\xi_{1}, \xi_{2}, \xi_{3}\right)=\psi_{1}\left(\xi_{1}\right) \psi_{0}\left(\xi_{2}\right) \psi_{0}\left(\xi_{3}\right)=\xi_{1}$ & \multirow{3}{*}{1} \\
\hline$\psi_{010}\left(\xi_{1}, \xi_{2}, \xi_{3}\right)=\psi_{0}\left(\xi_{1}\right) \psi_{1}\left(\xi_{2}\right) \psi_{0}\left(\xi_{3}\right)=\xi_{2}$ & \\
\hline$\psi_{001}\left(\xi_{1}, \xi_{2}, \xi_{3}\right)=\psi_{0}\left(\xi_{1}\right) \psi_{0}\left(\xi_{2}\right) \psi_{1}\left(\xi_{3}\right)=\xi_{3}$ & \\
\hline$\psi_{200}\left(\xi_{1}, \xi_{2}, \xi_{3}\right)=\psi_{2}\left(\xi_{1}\right) \psi_{0}\left(\xi_{2}\right) \psi_{0}\left(\xi_{3}\right)=\xi_{1}^{2}-1$ & \multirow{6}{*}{2} \\
\hline$\psi_{020}\left(\xi_{1}, \xi_{2}, \xi_{3}\right)=\psi_{0}\left(\xi_{1}\right) \psi_{2}\left(\xi_{2}\right) \psi_{0}\left(\xi_{3}\right)=\xi_{2}^{2}-1$ & \\
\hline$\psi_{002}\left(\xi_{1}, \xi_{2}, \xi_{3}\right)=\psi_{0}\left(\xi_{1}\right) \psi_{0}\left(\xi_{2}\right) \psi_{2}\left(\xi_{3}\right)=\xi_{3}^{2}-1$ & \\
\hline$\psi_{110}\left(\xi_{1}, \xi_{2}, \xi_{3}\right)=\psi_{1}\left(\xi_{1}\right) \psi_{1}\left(\xi_{2}\right) \psi_{0}\left(\xi_{3}\right)=\xi_{1} \xi_{2}$ & \\
\hline$\psi_{101}\left(\xi_{1}, \xi_{2}, \xi_{3}\right)=\psi_{1}\left(\xi_{1}\right) \psi_{0}\left(\xi_{2}\right) \psi_{1}\left(\xi_{3}\right)=\xi_{1} \xi_{3}$ & \\
\hline$\psi_{011}\left(\xi_{1}, \xi_{2}, \xi_{3}\right)=\psi_{0}\left(\xi_{1}\right) \psi_{1}\left(\xi_{2}\right) \psi_{1}\left(\xi_{3}\right)=\xi_{2} \xi_{3}$ & \\
\hline
\end{tabular}
Table I.

\section{B. The hyperbolic scheme for PC expansion}

The hyperbolic scheme restricts the combination of $\alpha_{1} \alpha_{2} \ldots \alpha_{\mathcal{K}}$ in the PC expansion to satisfy

$$
\left(\sum_{k=1}^{\mathcal{K}} \alpha_{k}^{q}\right)^{\frac{1}{q}} \leq r
$$

for $0<q \leq 1$, and thus the number of polynomial bases in the PC expansion in (2) can be reduced, based on $q$-quasinorm [21]. When $q$ equals 1 , (3) becomes the usual truncation scheme as shown in (1). When we set $q$ to 0.4 , the number of retained polynomial bases is reduced to 7 based on (3). The retained polynomial bases are presented in Table II for $q=1$ and $q=0.4$.

\begin{tabular}{c|c}
$q$ & $\alpha_{1} \alpha_{2} \alpha_{3}$ in $\psi_{\alpha_{1} \alpha_{2} \alpha_{3}}\left(\xi_{1}, \xi_{2}, \xi_{3}\right)$ \\
\hline 1 & $000,100,010,001,200,020,002,110,101,011$ \\
\hline 0.4 & $000,100,010,001,200,020,002$ \\
\hline \multicolumn{2}{c}{ TABLE II } \\
RETAINED POLYNOMIAL BASES WHEN $q=1$ AND $q=0.4$
\end{tabular}

\section{The least angle regression}

1) The linear regression model:

Assuming there are $\mathcal{K}$ Debye parameters of interest and $\mathcal{M}$ sets of input samples, we define the vector $\boldsymbol{\xi}=$ $\left\{\xi_{1}, \xi_{2}, \cdots, \xi_{\mathcal{K}}\right\}$ as the combination of these Debye parameters, and $\boldsymbol{\xi}^{(m)}$ as the $m$-th $\boldsymbol{\xi}$ for $1 \leq m \leq \mathcal{M}$. The LARS method is a classic model-selection method [21]. It aims to 
select the polynomial bases $\psi$ that highly correlate with the output of the linear regression model of

$$
\mathcal{T}\left(\boldsymbol{\xi}^{(m)}\right)=\sum_{l=1}^{\mathcal{L}} a_{\boldsymbol{\alpha}_{l}} \psi_{\boldsymbol{\alpha}_{l}}\left(\boldsymbol{\xi}^{(m)}\right)
$$

where $\mathcal{T}\left(\boldsymbol{\xi}^{(m)}\right)$ is the predicted value of $|\boldsymbol{E}|^{2}$ for $\boldsymbol{\xi}^{(m)}$, the polynomial term $a_{\boldsymbol{\alpha}_{l}} \psi_{\boldsymbol{\alpha}_{l}}\left(\boldsymbol{\xi}^{(m)}\right)$ is called a predictor of $\mathcal{T}\left(\boldsymbol{\xi}^{(m)}\right), \boldsymbol{\alpha}_{l}$ indicates the index of the $l$-th $\psi$, and $a$ is the coefficient of $\psi$.

Let $\boldsymbol{a}$ be a vector which consists of $\mathcal{L}$ coefficients as in $\boldsymbol{a}=\left\{a_{\boldsymbol{\alpha}_{1}}, \cdots, a_{\boldsymbol{\alpha}_{\mathcal{L}}}\right\}^{T}, \widehat{\mathcal{T}}\left(\boldsymbol{\xi}^{(m)}\right)$ be the current LARS update of $\mathcal{T}\left(\boldsymbol{\xi}^{(m)}\right)$ and the vector $\widehat{\mathcal{T}}=\left\{\widehat{\mathcal{T}}\left(\boldsymbol{\xi}^{(1)}\right), \cdots, \widehat{\mathcal{T}}\left(\boldsymbol{\xi}^{(\mathcal{M})}\right)\right\}^{T}$. The matrix $\boldsymbol{\Psi}=\left(\psi_{\boldsymbol{\alpha}_{l}}\left(\boldsymbol{\xi}^{(m)}\right), m=1 \sim \mathcal{M}, l=1 \sim \mathcal{L}\right)$ formed by the polynomial bases is presented as

$$
\boldsymbol{\Psi}=\left[\begin{array}{cccc}
\psi_{\boldsymbol{\alpha}_{1}}\left(\boldsymbol{\xi}^{(1)}\right) & \psi \boldsymbol{\alpha}_{2}\left(\boldsymbol{\xi}^{(1)}\right) & \ldots & \psi \boldsymbol{\alpha}_{\mathcal{L}}\left(\boldsymbol{\xi}^{(1)}\right) \\
\psi_{\boldsymbol{\alpha}_{1}}\left(\boldsymbol{\xi}^{(2)}\right) & \psi_{\boldsymbol{\alpha}_{2}}\left(\boldsymbol{\xi}^{(2)}\right) & \ldots & \psi \boldsymbol{\alpha}_{\mathcal{L}}\left(\boldsymbol{\xi}^{(2)}\right) \\
\vdots & \vdots & \ddots & \vdots \\
\psi \boldsymbol{\alpha}_{1}\left(\boldsymbol{\xi}^{(\mathcal{M})}\right) & \psi_{\boldsymbol{\alpha}_{2}}\left(\boldsymbol{\xi}^{(\mathcal{M})}\right) & \ldots & \psi_{\boldsymbol{\alpha}_{\mathcal{L}}}\left(\boldsymbol{\xi}^{(\mathcal{M})}\right)
\end{array}\right]
$$

2) The LARS iteration:

At the start of the LARS method, $\boldsymbol{a}$ is initialised to $\mathbf{0}$. Let $\mathcal{C}=$ $\left\{\mathcal{C}_{1}, \mathcal{C}_{2}, \cdots, \mathcal{C}_{\mathcal{L}}\right\}^{T}$ be the vector of the correlation between $\psi$ and $(\mathcal{E}-\widehat{\mathcal{T}})$ calculated as in

$$
\mathcal{C}=\Psi^{T}(\mathcal{E}-\widehat{\mathcal{T}})
$$

where $\mathcal{E}=\left\{\left|\boldsymbol{E}_{1}\right|^{2}, \cdots,\left|\boldsymbol{E}_{\mathcal{M}}\right|^{2}\right\}^{T}$ is a vector of the $\mathcal{M}|\boldsymbol{E}|^{2}$ obtained from the FDTD simulations, and $\left|\boldsymbol{E}_{m}\right|$ is $|\boldsymbol{E}|$ when $\boldsymbol{\xi}^{(m)}$ is used for the FDTD computation and the difference between $\mathcal{E}$ and $\widehat{\mathcal{T}}$ is called the residual vector. The value of $\widehat{\mathcal{T}}\left(\boldsymbol{\xi}^{(m)}\right)$ is also initialised to zero at the start of LARS method. Each element in $\mathcal{C}$ such as $\mathcal{C}_{l}$ refers to the correlation between $\psi_{\boldsymbol{\alpha}_{l}}$ and the residual vector. When $\widetilde{k}$ satisfies

$$
\left|\mathcal{C}_{\widetilde{k}}\right|=\max _{1 \leq l \leq \mathcal{L}}\left|\mathcal{C}_{l}\right|
$$

the $\psi_{\boldsymbol{\alpha}_{\tilde{k}}}$, which has the highest correlation with the residual vector, and its corresponding coefficient $a_{\boldsymbol{\alpha}_{\tilde{k}}}$ are chosen as a predictor of $\mathcal{T}(\boldsymbol{\xi})$. We put the chosen $a_{\boldsymbol{\alpha}_{\tilde{k}}} \psi_{\boldsymbol{\alpha}_{\tilde{k}}}$ into a set $\mathcal{Q}$ as in $\mathcal{Q}=\left\{a_{\boldsymbol{\alpha}_{\tilde{k}}} \psi_{\boldsymbol{\alpha}_{\tilde{k}}}\right\}$ and put $\widetilde{k}$ into a set $\mathcal{A}$ as in $\mathcal{A}=$ $\{\widetilde{k}, \widetilde{k} \in[1, \mathcal{L}]\}$, where the number of elements in $\mathcal{A}$ increases with the iteration. A matrix $\hat{\boldsymbol{\Psi}}$ represents the chosen $\psi$ as in $\hat{\boldsymbol{\Psi}}=\left(\psi_{\boldsymbol{\alpha}_{\widetilde{k}}}\left(\boldsymbol{\xi}^{(m)}\right), m=1 \sim \mathcal{M}, \widetilde{k} \in[1, \mathcal{L}]\right)$ and a vector $\hat{\boldsymbol{a}}$ indicates the coefficients of the chosen $\psi$ as in $\hat{\boldsymbol{a}}=\left\{a_{\boldsymbol{\alpha}_{\tilde{k}}}, \widetilde{k} \in\right.$ $[1, \mathcal{L}]\}^{T}$. At the first LARS iteration, for example, when $\widetilde{k}=6$, we form $\hat{\boldsymbol{\Psi}}=\left\{\psi_{\boldsymbol{\alpha}_{6}}\left(\boldsymbol{\xi}^{(1)}\right), \cdots, \psi_{\boldsymbol{\alpha}_{6}}\left(\boldsymbol{\xi}^{(\mathcal{M})}\right)\right\}^{T}$ from Table I and calculate $\hat{\boldsymbol{a}}$ as in

$$
\hat{\boldsymbol{a}}=\left(\hat{\boldsymbol{\Psi}}^{T} \hat{\boldsymbol{\Psi}}\right)^{-1} \hat{\boldsymbol{\Psi}}^{T} \mathcal{E} .
$$

As $\hat{\boldsymbol{a}}=\left\{a_{\boldsymbol{\alpha}_{6}}\right\}^{T}$, (7) practically calculates the value of $a_{\boldsymbol{\alpha}_{6}}$ and $\boldsymbol{a}$ thus becomes $\left\{0, \cdots, 0, a_{\boldsymbol{\alpha}_{6}}, 0, \cdots, 0\right\}^{T} \cdot \widehat{\mathcal{T}}\left(\boldsymbol{\xi}^{(m)}\right)$ is updated as in

$$
\widehat{\mathcal{T}}^{(w+1)}\left(\boldsymbol{\xi}^{(m)}\right)=\widehat{\mathcal{T}}^{(w)}\left(\boldsymbol{\xi}^{(m)}\right)+\hat{\gamma}_{\widetilde{k}^{(w)}} a_{\boldsymbol{\alpha}_{\widetilde{k}^{(w)}}} \psi_{\boldsymbol{\alpha}_{\widetilde{k}^{(w)}}},
$$

where $\widehat{\mathcal{T}}^{(w)}\left(\boldsymbol{\xi}^{(m)}\right)$ and $\widetilde{k}^{(w)}$ are $\widehat{\mathcal{T}}\left(\boldsymbol{\xi}^{(m)}\right)$ and $\widetilde{k}$ at $w$-th LARS iteration and $\hat{\gamma}_{\widetilde{k}}$ indicates the update coefficient of the LARS method corresponding to the $\widetilde{k}$-th polynomial basis. $\hat{\gamma}_{\widetilde{k}}$ can be calculated by

$$
\hat{\gamma}_{\widetilde{k}}=\min ^{+}\left\{\frac{\left|\mathcal{C}_{\widetilde{k}}\right|-\mathcal{C}_{\widetilde{k}}}{A_{\mathcal{A}}-a_{\boldsymbol{\alpha}_{\widetilde{k}}}}, \frac{\left|\mathcal{C}_{\widetilde{k}}\right|+\mathcal{C}_{\widetilde{k}}}{A_{\mathcal{A}}+a_{\boldsymbol{\alpha}_{\widetilde{k}}}}\right\},
$$

where "min ${ }^{+}$" indicates that $\hat{\gamma}_{\widetilde{k}}$ only takes the positive minimum component in (9) and $A_{\mathcal{A}}$ is given by

$$
A_{\mathcal{A}}=\left(1_{\mathcal{A}}^{T} \hat{\mathbf{\Psi}}^{T} \hat{\boldsymbol{\Psi}} 1_{\mathcal{A}}\right)^{-\frac{1}{2}}
$$

where $1_{\mathcal{A}}$ is a vector of 1 of length equaling the size of $\mathcal{A}$ as in $1_{\mathcal{A}}=\{1,1, \cdots, 1\}^{T}$. When $\mathcal{A}$ includes one element $\mathcal{A}=\{6\}, 1_{\mathcal{A}}$ equals $1_{\mathcal{A}}=\{1\}^{T}$. In the successive iteration, $\widetilde{k}^{(w)}$ meets (6) and $\widetilde{k}^{(w)} \neq \widetilde{k}^{\left(w^{\dagger}\right)}$ where $1 \leq w^{\dagger}<w$.

Such a process of determining a predictor, adding it into $\mathcal{Q}$, and updating $\widehat{\mathcal{T}}\left(\boldsymbol{\xi}^{(m)}\right)$ is called a LARS iteration. At each LARS iteration, one non-zero form of $a_{\boldsymbol{\alpha}_{l}} \psi_{\boldsymbol{\alpha}_{l}}$ is included into $\mathcal{Q}$. As a result, the number of elements in $\mathcal{Q}$ is the same as the LARS iteration number. The maximum number of the LARS iteration equals $\mathcal{L}$, in which case all $\psi$ are incorporated in $\mathcal{Q}$.

\section{3) The leave-one-out cross-validation method:}

After each LARS iteration, we calculate $\mathcal{T}\left(\boldsymbol{\xi}^{(m)}\right)$ in (4) and use the leave-one-out cross-validation (LOOCV) method [26] to evaluate the accuracy of $\mathcal{T}\left(\boldsymbol{\xi}^{(m)}\right)$. The leave-one-out (LOO) error $\varepsilon_{l}$ of $\mathcal{T}\left(\boldsymbol{\xi}^{(m)}\right)$ quantifies its accuracy as in

$$
\varepsilon_{l}=\frac{1}{\mathcal{M}} \sum_{m=1}^{\mathcal{M}}\left(\frac{\left|\boldsymbol{E}_{m}\right|^{2}-\mathcal{T}\left(\boldsymbol{\xi}^{(m)}\right)}{1-h_{m}}\right)^{2},
$$

where $h_{m}$ is the $m$-th diagonal element of the square matrix $\hat{\boldsymbol{\Psi}}\left(\hat{\boldsymbol{\Psi}}^{T} \hat{\boldsymbol{\Psi}}\right)^{-1} \hat{\boldsymbol{\Psi}}^{T}$. Note that we use $\mathcal{T}\left(\boldsymbol{\xi}^{(m)}\right)$, not $\widehat{\mathcal{T}}\left(\boldsymbol{\xi}^{(m)}\right)$, to calculate $\varepsilon_{l}$. They have different meanings in the LARS method. $\widehat{\mathcal{T}}\left(\boldsymbol{\xi}^{(m)}\right)$ enables us to select a set of predictors out of the $\mathcal{L}$ predictors, whereas $\mathcal{T}\left(\boldsymbol{\xi}^{(m)}\right)$ is used as the estimate of $\left|\boldsymbol{E}_{m}\right|^{2}$ for $1 \leq m \leq \mathcal{M}$.

We control the LARS procedure by setting a threshold named as target error $\varepsilon_{t}$ and calculate $\varepsilon_{l}$ after each LARS iteration, and then compare $\varepsilon_{l}$ with $\varepsilon_{t}$. When $\varepsilon_{l}$ is less than or equal to $\varepsilon_{t}$, the LARS procedure is terminated. The termination of the LARS procedure may happen before all $\psi$ are selected.

\section{The adaptive LARS method}

\section{1) The potential problems of the LARS method:}

Comparing with other model selection methods such as the least absolute shrinkage and selection operator (LASSO) method [27], the LARS method is more stable and computationally efficient. Nevertheless, there are some factors that may negatively influence the performance of the LARS method with respect to the accuracy and the stability.

1) The LARS method fails to capture the relations between $\boldsymbol{\xi}^{(m)}$ and $\mathcal{T}\left(\boldsymbol{\xi}^{(m)}\right)$. 
When the number of input samples is limited, the LARS method may not be able to capture the relations between $\boldsymbol{\xi}^{(m)}$ and $\mathcal{T}\left(\boldsymbol{\xi}^{(m)}\right)$. This phenomenon is called underfitting. When underfitting occurs, $\mathcal{T}\left(\boldsymbol{\xi}^{(m)}\right)$ usually shows a low accuracy.

2) Some chosen samples negatively impact the accuracy of $\mathcal{T}\left(\boldsymbol{\xi}^{(m)}\right)$.

It is possible that some chosen $\boldsymbol{\xi}$ yields values of $|\boldsymbol{E}|^{2}$ which are substantially larger or smaller than the $|\boldsymbol{E}|^{2}$ values corresponding to all other $\boldsymbol{\xi}$. This sort of $|\boldsymbol{E}|^{2}$ is called an outlier which may negatively impact the accuracy of $\mathcal{T}\left(\boldsymbol{\xi}^{(m)}\right)$.

3) Unreasonable setting of $\varepsilon_{t}$.

In the LARS method, we calculate $\varepsilon_{l}$ in (11) after each LARS iteration and stop the LARS procedure when $\varepsilon_{l}$ is less than $\varepsilon_{t}$ or the number of LARS iterations equals $\mathcal{L}$. The setting of $\varepsilon_{t}$ exerts a crucial influence on the final result of LARS method. An unreasonable setting of $\varepsilon_{t}$ may cause unsatisfactory results. When $\varepsilon_{l}$ is higher than $\varepsilon_{t}$ throughout all the LARS iterations, the LARS procedure does not stop until all $\psi_{\boldsymbol{\alpha}_{l}}$ are incorporated into $\mathcal{Q}$. In such cases, the optimal result, which is most close to the result of MCM, may have already appeared in the earlier LARS iterations. However, due to the high value of $\varepsilon_{l}$, the LARS procedure does not stop at this iteration. Similarly, it is possible that the LARS procedure stops too early when $\varepsilon_{l}$ is less than $\varepsilon_{t}$ after a few number of LARS iterations. As a result, only a limited number of $\psi_{\boldsymbol{\alpha}_{l}}$ participate in the calculation of $\mathcal{T}(\boldsymbol{\xi})$ and thus the estimated standard deviation becomes unreliable.

4) Lack of flexibility on the required number of input samples.

To successfully perform the matrix inversion in (10), the total number of the chosen $a$ should not exceed $\mathcal{M}$. In the LARS method, $\mathcal{M}$ should satisfy $\mathcal{L} \leq \mathcal{M}$. Such a condition restricts the minimum number of input samples.

2) Methods to improve the LARS method:

Four techniques are proposed aiming to circumvent these obstacles. In this paper, the LARS method with these four techniques is called as the adaptive LARS method.

1) In the LARS method, we can observe the possible underfitting phenomenon by detecting the change of $\varepsilon_{l}$. If $\varepsilon_{l}$ obtained at the $w$-th LARS iteration is higher than the $(w-1)$ th $\varepsilon_{l}$, the underfitting situation might have occurred. We prevent underfitting by stopping the LARS procedure if $\varepsilon_{l}$ of the $w$-th LARS iteration exceeds that of the $(w-1)$ th LARS iteration by a certain threshold value and taking the chosen polynomial bases obtained at the $(w-1)$ th LARS iteration as the final chosen polynomial bases.

2) We set the minimum number of the LARS iterations to prevent the LARS procedure from being terminated too early by

a) Setting the maximum number of the LARS itera- tions to $\mathcal{L}$.

b) Reducing $\varepsilon_{t}$ by a certain percentage at each LARS iteration when $\varepsilon_{l}$ is less than $\varepsilon_{t}$ before $\zeta$ LARS iterations so as to continue the LARS procedure until at least $\zeta$ polynomial bases are included in $\mathcal{Q}$, where $\zeta$ is a certain number of the LARS iterations for $1 \leq \zeta<\mathcal{L}$.

3) In the LARS method, we obtain $\mathcal{M}|\boldsymbol{E}|^{2}$ from the $\mathcal{M}$ FDTD simulations. The variance of the $\mathcal{M}|\boldsymbol{E}|^{2}$ is called sample variance. Since the LHS method has the property of spreading the sample points evenly across all possible values, it is likely that the sample variance obtained by LHS method is able to reflect the population variance to a certain degree [28]. We make use of this feature as an auxiliary condition to help us optimise the LARS result by taking the following steps.

a) Calculate the sample variance $\hat{\sigma}^{2}$ of $\mathcal{M}|\boldsymbol{E}|^{2}$ in

$$
\hat{\sigma}^{2}=\frac{1}{\mathcal{M}-1} \sum_{m=1}^{\mathcal{M}}\left(\left|\boldsymbol{E}_{m}\right|^{2}-\frac{1}{\mathcal{M}} \sum_{i=1}^{\mathcal{M}}\left|\boldsymbol{E}_{i}\right|^{2}\right)^{2} \text {. }
$$

b) Calculate $\varepsilon_{l}$ at each LARS iteration. When $\varepsilon_{l}$ is less than $\varepsilon_{t}$ and the number of LARS iterations is larger than $\zeta$ and less than $\varsigma$, we run further $\iota$ LARS iterations instead of stopping LARS procedure, where $\iota \leq(\mathcal{L}-\varsigma)$ and $\zeta<\varsigma<\mathcal{L}$. When $\varepsilon_{l} \leq \varepsilon_{t}$, the variance $\left(\sigma^{(\mathcal{M})}\right)^{2}$ is calculated by

$$
\left(\sigma^{(\mathcal{M})}\right)^{2}=\left\{\sum_{\mathcal{Q}} a_{\boldsymbol{\alpha}_{l}}{ }^{2}, 1 \leq l \leq \mathcal{L}\right\}
$$

at the end of each LARS iteration.

c) Compare the sample variance in (12) with the variance calculated at each LARS iteration in (13). We consider $\left(\sigma^{(\mathcal{M})}\right)^{2}$ closest to $\hat{\sigma}^{2}$ as the final result.

4) The restriction of $\mathcal{L} \leq \mathcal{M}$ is lifted by applying the L2 regularisation scheme to the LARS method. This enables the LARS method to get a reasonable pseudoinverse by introducing a regularisation factor $\varrho$ into (10). Therefore, when the number of the chosen $a$ is larger than $\mathcal{M}$, we calculate $A_{\mathcal{A}}$ by

$$
A_{\mathcal{A}}=\left(1_{\mathcal{A}}^{T} \hat{\mathbf{\Psi}}^{T} \hat{\mathbf{\Psi}} 1_{\mathcal{A}}+1_{\mathcal{A}}^{T} \varrho 1_{\mathcal{A}}\right)^{-\frac{1}{2}} .
$$

With the L2 regularisation scheme, the LARS method is capable of functioning even when $\mathcal{M}<\mathcal{L}$. Such feature of the L2 regularisation scheme is used to identify the optimal value of $\mathcal{M}$ by running the LARS method varying $\mathcal{M}$ from 1 to a certain number $(\leq \mathcal{L}) . \mathcal{M}$ is determined when $\sigma^{(\mathcal{M})}$ in (13) reaches the stable status. When $\vartheta=\frac{\left|\sigma^{(\mathcal{M}-1)}-\sigma^{(\mathcal{M}-2)}\right|}{\sigma^{(\mathcal{M}-1)}}$ and $\vartheta^{\dagger}=\frac{\left|\sigma^{(\mathcal{M})}-\sigma^{(\mathcal{M}-1)}\right|}{\sigma^{(\mathcal{M})}}$ are less than a certain small value $\varpi, \sigma^{(\mathcal{M})}$ has no obvious change with the increase of $\mathcal{M}$, and thus $\sigma^{(\mathcal{M})}$ can be regarded as stable, and $\mathcal{M}$ at this point is regarded as the optimal.

The adaptive LARS method is summarised in Fig. 1. 


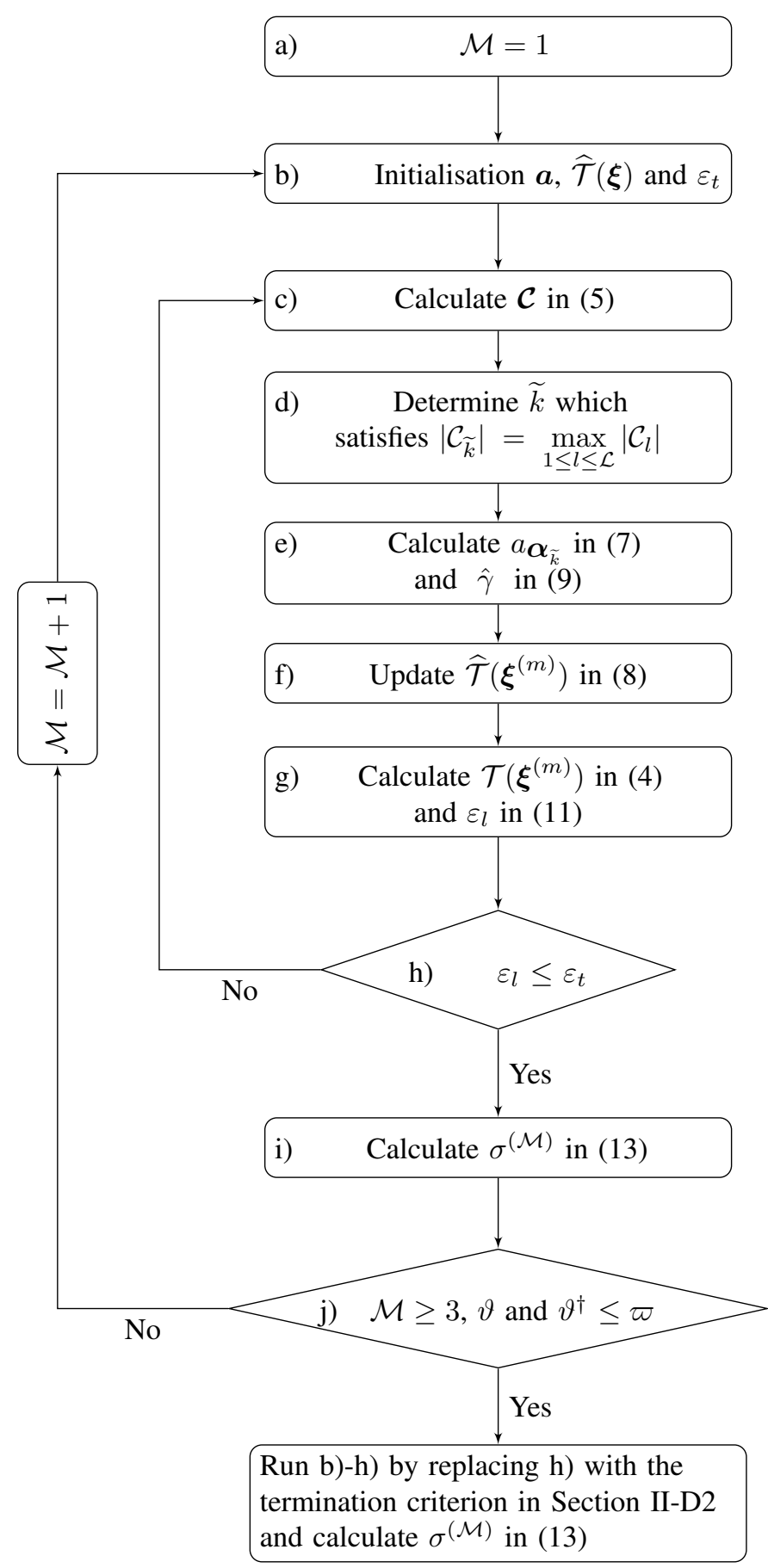

Fig. 1. The flow chart of the adaptive LARS method

\section{NUMERICAL EXPERIMENTS SETTING FOR UNCERTAINTY QUANTIFICATION}

The MCM, the LARS method and the adaptive LARS method are utilised to quantify the uncertainty of the FDTD results.

\section{A. Radio environment setting}

In the FDTD space, we place a point soft source excitation outside the human body and observe $\boldsymbol{E}$ in the middle of the prostate tissue. 10 layers of the complex frequency shiftedperfect matched layers (CFS-PML) [29] [30] are utilised to terminate the FDTD space. The DHP used in this work is $1 \mathrm{~mm}$ spatial resolution and provided by RIKEN (Saitama, Japan) under the non-disclosure agreement between RIKEN and the University of Manchester. The usage was approved by RIKEN ethical committee.

This simulation scenario is depicted in Fig. 2. The excitation location is $17 \mathrm{~mm}$ away from the human body and the observation location is in the middle of the prostate tissue.

There are five influential tissues (fat, skin, muscle, bone and prostate) between the excitation and observation. $|\boldsymbol{E}|^{2}$ observed changes significantly when one or more of the Debye parameters of the influential tissues are changed. In the FDTD calculations, the relationship between $\boldsymbol{E}$ and the electric flux density $\boldsymbol{D}$ in one-pole Debye model is written as

$$
\boldsymbol{D}=\epsilon_{0}\left[\epsilon_{\infty}+\frac{\Delta \epsilon}{1+\jmath \omega \tau}+\frac{\kappa_{S}}{\jmath \omega \epsilon_{0}}\right] \boldsymbol{E}
$$

where $\epsilon_{0}$ is the permittivity of a vacuum $\left(8.8541 \times 10^{-12}\right.$ $\mathrm{F} / \mathrm{m}), \omega$ is the angular frequency, $\jmath$ is the imaginary unit satisfying $\jmath=\sqrt{-1}, \kappa_{S}$ is the static conductivity, $\epsilon_{\infty}$ is the optical relative permittivity, $\epsilon_{S}$ is the static relative permittivity, $\tau$ is the relaxation time, and $\Delta \epsilon$ satisfies $\Delta \epsilon=\epsilon_{S}-\epsilon_{\infty}$. Each tissue in the DHP is associated with four Debye parameters $\left(\kappa_{S}, \epsilon_{\infty}, \Delta \epsilon, \tau\right)$. The Debye parameters for all human tissues are presented in [31]. The $\epsilon_{\infty}$ and $\Delta \epsilon$ are the influential Debye parameters for the five influential tissues.

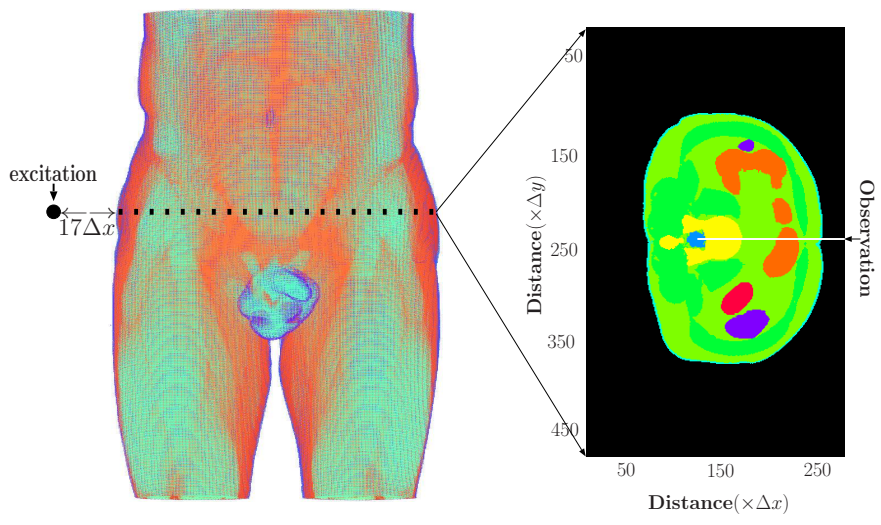

Fig. 2. Numerical simulation setup.

\section{B. Numerical experiments for uncertainty quantification}

1) The Monte Carlo method

a) Generate the $10^{4}$ random $\epsilon_{\infty}$ and $\Delta \epsilon$ for each of 5 influential tissues yielding the normal distribution. In our scenario, $10^{4}$ input samples enable the MCM method to reach convergence. $\mathcal{K}(=10)$ variables and their average and the corresponding standard deviation are listed in Table III. When we generate $10^{4}$ samples for each variable, the relative permittivity and the conductivity of each tissue vary within \pm 10 percent.

b) Randomly pick one sample out of $10^{4}$ samples for each variable and combine these chosen samples 


\begin{tabular}{|l|l|c|c|}
\hline$\xi$ & Meaning of $\xi$ & Average & Standard deviation \\
\hline$\xi_{1}$ & $\epsilon_{\infty}$ of fat & 6.80 & 0.68 \\
\hline$\xi_{2}$ & $\Delta \epsilon$ of fat & 7.37 & 0.74 \\
\hline$\xi_{3}$ & $\epsilon_{\infty}$ of skin & 18.07 & 1.81 \\
\hline$\xi_{4}$ & $\Delta \epsilon$ of skin & 29.87 & 2.99 \\
\hline$\xi_{5}$ & $\epsilon_{\infty}$ of muscle & 28.93 & 2.88 \\
\hline$\xi_{6}$ & $\Delta \epsilon$ of muscle & 28.02 & 2.81 \\
\hline$\xi_{7}$ & $\epsilon_{\infty}$ of bone & 1.53 & 0.15 \\
\hline$\xi_{8}$ & $\Delta \epsilon$ of bone & 4.01 & 0.40 \\
\hline$\xi_{9}$ & $\epsilon_{\infty}$ of prostate & 32.82 & 3.28 \\
\hline$\xi_{10}$ & $\Delta \epsilon$ of prostate & 27.73 & 2.78 \\
\hline
\end{tabular}

THE AVERAGE VALUES AND STANDARD DEVIATIONS OF THE 10 VARIABLES AND THEIR CORRESPONDING NOTATION

to produce 1 combination of the 10 variables as in $\boldsymbol{\xi}=\left\{\xi_{1}, \xi_{2}, \cdots, \xi_{10}\right\}$.

c) Repeat Step $1 \mathrm{~b} 10^{4}$ times to produce $10^{4} \boldsymbol{\xi}$. In Step $1 \mathrm{~b}$, we do not choose the same samples as those chosen earlier.

d) Run $10^{4}$ FDTD simulations each with a different $\boldsymbol{\xi}$ to obtain $10^{4}|\boldsymbol{E}|^{2}$.

e) Calculate

$$
\nu(\mathcal{M})=\frac{1}{\mathcal{M}} \sum_{m=1}^{\mathcal{M}}\left|\boldsymbol{E}_{m}\right|^{2}
$$

and

$$
\sigma(\mathcal{M})^{2}=\frac{1}{\mathcal{M}-1} \sum_{m=1}^{\mathcal{M}}\left(\left|\boldsymbol{E}_{m}\right|^{2}-\nu(\mathcal{M})\right)^{2}
$$

of the first $\mathcal{M}|\boldsymbol{E}|^{2}$ among the $10^{4}|\boldsymbol{E}|^{2}$ varying $\mathcal{M}$ from 2 to $10^{4}$

2) The LARS method

In the LARS method, the setting of $\varepsilon_{t}$ is crucial. Empirically, $\varepsilon_{t}=10^{-3}$ is a reasonable value in our scenario enabling the LARS method to have a stable performance. Fig. 3 and Fig. 4 show the $\sigma^{(\mathcal{M})}$ and the $\varepsilon_{l}$ varying LARS iterations respectively in case when $q=0.4, \mathcal{K}=10, \mathcal{M}=20$ and $\mathcal{L}=20$. The $\varepsilon_{l}$ is less than $\varepsilon_{t}$ at the 14th LARS iteration and the LARS procedure is therefore terminated at the 14th iteration. Thus, there are $14 a_{\boldsymbol{\alpha}_{l}} \psi_{\boldsymbol{\alpha}_{l}}$ in total in $\mathcal{Q}$, in the order of $l=\{6,14,7,3,1,2,20,11,13,9,4,8,12,19\}$.

We set $\varrho$ in (14) to $10^{-8}$ in order to obtain an accurate pseudoinverse. In the case of 10 Debye parameters of interest as shown in Table III, $\sigma^{(\mathcal{M})}$ is calculated in (13) when $q=0.4, r=2$, and $\varepsilon_{t}=10^{-3}$.

3) The adaptive LARS method

The same parameters as the LARS method are used for the adaptive LARS method. In the adaptive LARS method, the LARS method is run first to determine the optimal value of $\mathcal{M}$. We determine the optimal $\mathcal{M}$ under the condition that $\vartheta$ and $\vartheta^{\dagger}$ are less than $\varpi$. The setting of $\varpi$ is application-specific. In our scenario, $\varpi$ of 0.03 enables us to identify the stable $\sigma^{(\mathcal{M})}$. The condition of $\vartheta$ and $\vartheta^{\dagger}$ less than 0.03 is satisfied when $\mathcal{M}=15$.

Fig. 5 shows $\sigma^{(\mathcal{M})}$ varying $\mathcal{M}$ from 1 to $20(=\mathcal{L})$. $\sigma\left(10^{4}\right)$ in (17) from $10^{4}$ FDTD results in MCM is presented as a dashed line for comparison. The results of

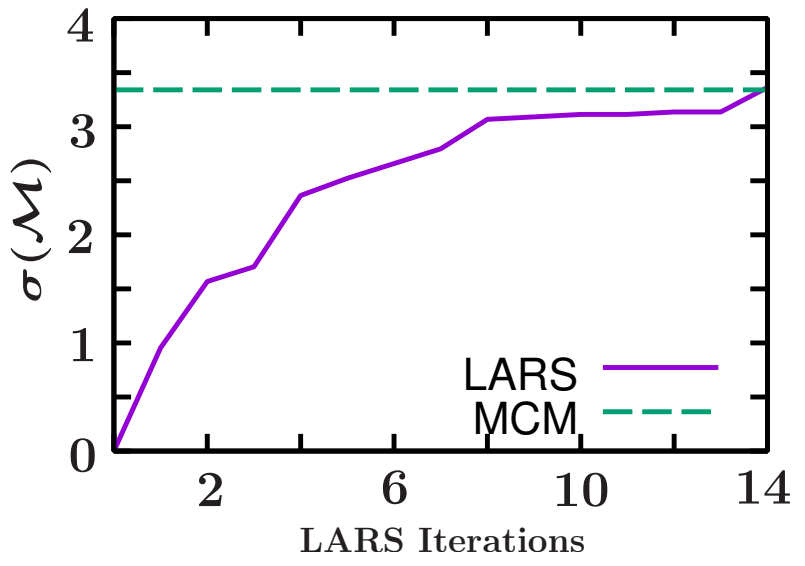

Fig. 3. $\sigma^{(\mathcal{M})}$ in (13) from the LARS method and $\sigma\left(10^{4}\right)$ in (17) from MCM is presented as a dashed line for comparison.

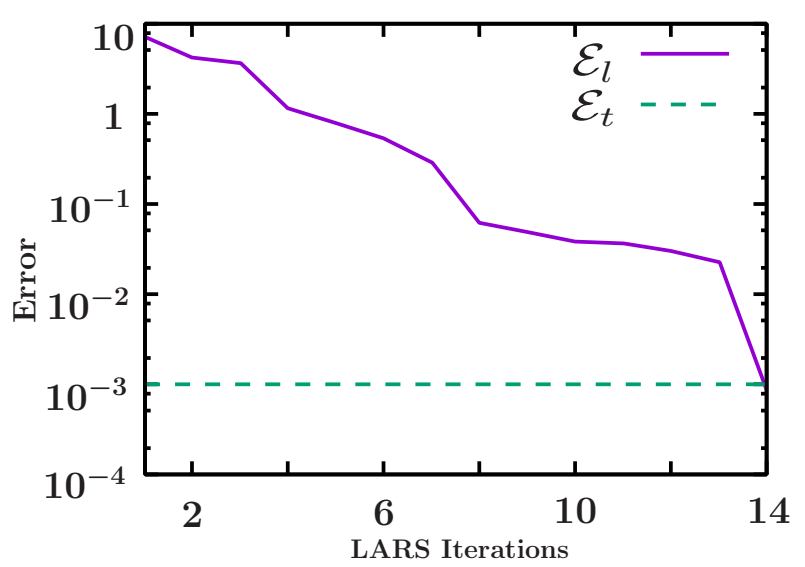

Fig. 4. The LOO error varying the LARS iterations.

$\sigma^{(\mathcal{M})}$ are neither stable nor accurate when $\mathcal{M}<15$ and the accuracy of $\sigma^{(\mathcal{M})}$ shows no obvious improvement when $\mathcal{M} \geq 15$. This proves that $\mathcal{M}=15$ is a optimal value in our scenario. We use 15 samples for UQ in the adaptive LARS methods leading to the adaptive LARS method which only requires $75 \%$ of the FDTD simulations needed by the original LARS method. We run the LARS iteration again with the determined $\mathcal{M}$ until one of the conditions which is described in Table IV is triggered. Table IV lists the conditions which may happen in the LARS procedure and the corresponding steps to calculate $\sigma^{(\mathcal{M})}$ of $|\boldsymbol{E}|^{2}$. Empirically, we set $\zeta=\frac{\mathcal{L}}{4}, \iota=\frac{5 \mathcal{L}}{6}$, and $\varsigma=\frac{\mathcal{L}}{6}$.

The adaptive LARS method mainly focuses on determining $\sigma^{(\mathcal{M})}$. The calculation of $\nu$ is identical for both the adaptive LARS method and the LARS method. We calculate $\nu$ in (16) with the determined $\mathcal{M}$. 


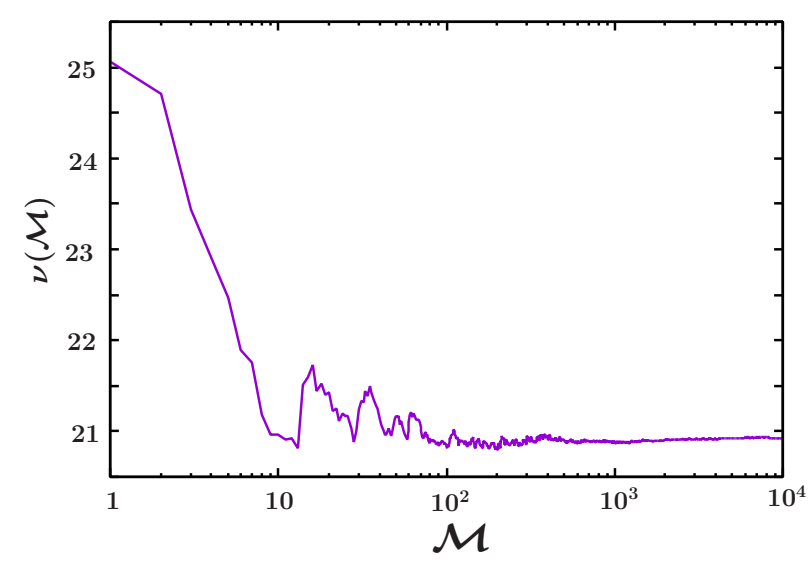

Fig. 6. $\nu(\mathcal{M})$ in (16) from MCM.

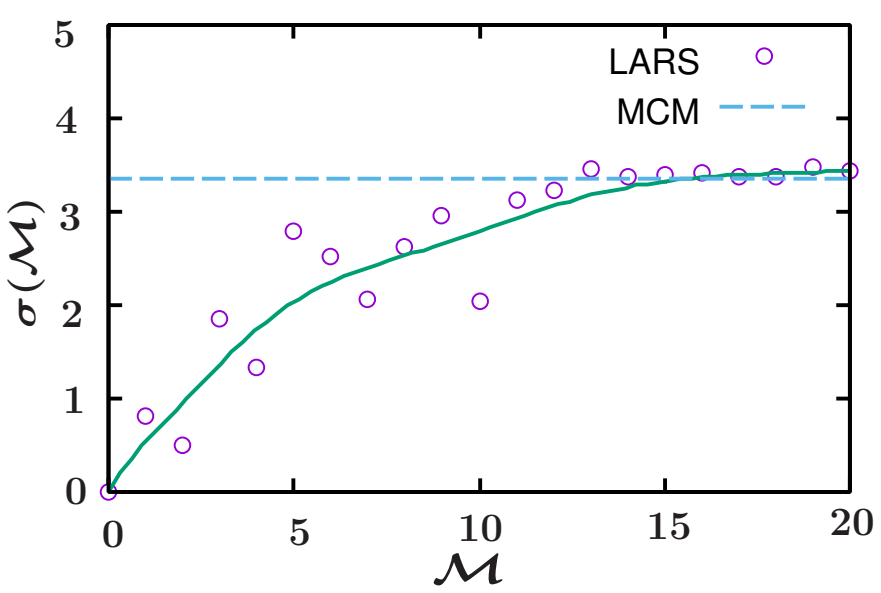

Fig. 5. $\sigma^{(\mathcal{M})}$ in (13) from the LARS method varying $\mathcal{M}$ from 0 to 20 . $\sigma\left(10^{4}\right)$ in (17) from MCM is presented as a dashed line for comparison purpose.

\section{RESULTS AND DISCUSSIONS}

We show $\nu$ and $\sigma^{(\mathcal{M})}$ obtained from the MCM, the LARS and the adaptive LARS methods. Some results obtained from the LARS method are not satisfactory. We present these results and their corresponding results obtained from the adaptive LARS method as a demonstration of the merit of the adaptive LARS method.

\section{A. Results}

1) Average values

The $\nu(\mathcal{M})$ of the first $\mathcal{M}|\boldsymbol{E}|^{2}$ obtained from the MCM is presented in Fig. 6, where $\mathcal{M}$ varies from 2 to $10^{4}$. Fig. 7 shows $\nu$ obtained from the LARS method when $\mathcal{M}=15, \mathcal{K}=10$ and $r=2$. The $\nu\left(10^{4}\right)$ from the MCM is presented as a straight line for comparison.

2) Standard deviations

Fig. 8 is $\sigma(\mathcal{M})$ in (17) of the first $\mathcal{M}|\boldsymbol{E}|^{2}$ obtained from the MCM, where $\mathcal{M}$ varies from 2 to $10^{4}$.

Fig. 9 presents $\sigma^{(\mathcal{M})}$ obtained from the LARS method when $\mathcal{M}=15, \mathcal{K}=10$ and $r=2 . \sigma\left(10^{4}\right)$ from the MCM is presented as a straight line for comparison. We call the results of the LARS method more than \pm

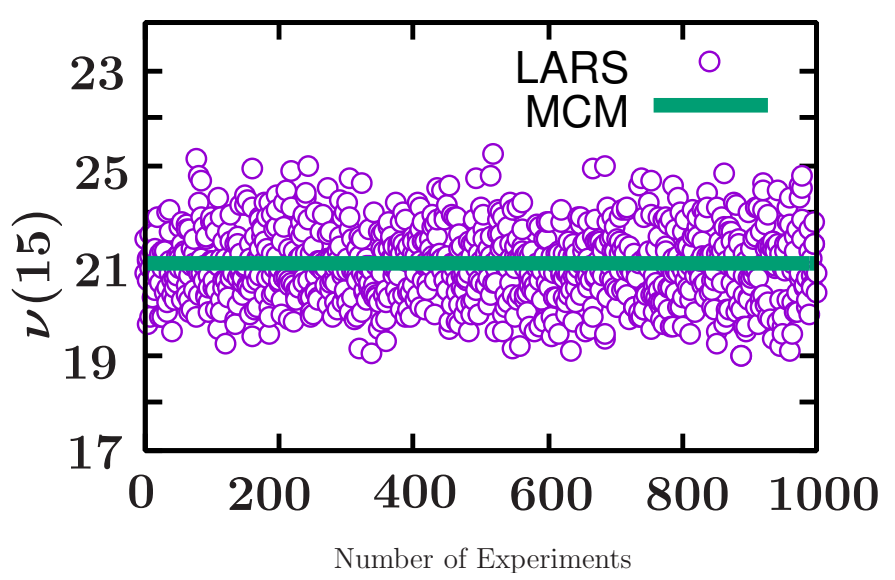

Fig. 7. $1000 \nu(15)$ from the LARS method and $\nu\left(10^{4}\right)$ from MCM.

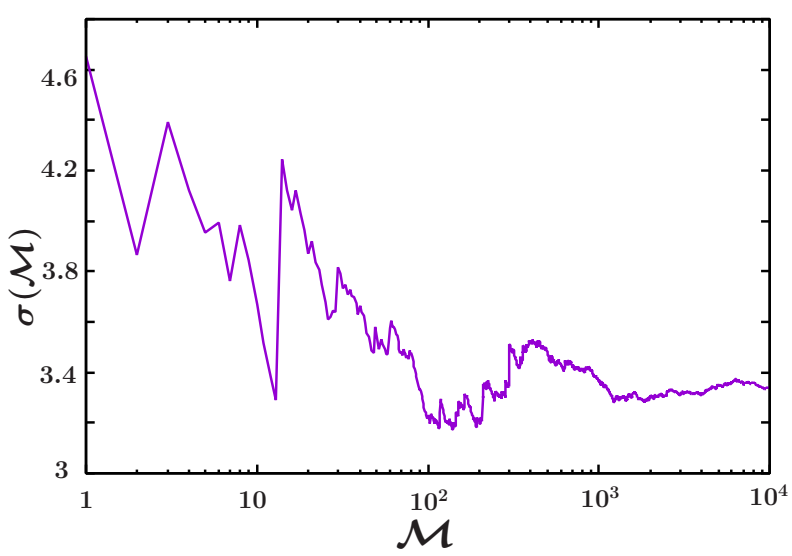

Fig. 8. $\sigma(\mathcal{M})$ in (17) from the MCM.

5\% away from the result of MCM as unsatisfactory as shown in Fig. 10a. These unsatisfactory results are then improved by the adaptive LARS method as shown in Fig. 10b.

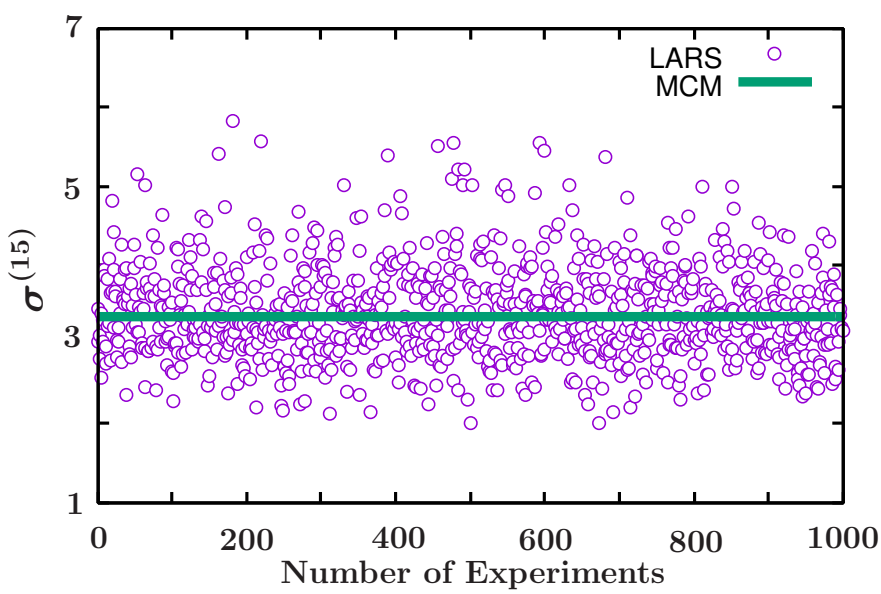

Fig. 9. $1000 \sigma^{(15)}$ from the LARS method and $\sigma\left(10^{4}\right)$ in (17) from MCM. 


\begin{tabular}{|c|c|}
\hline Conditions & Steps to calculate $\sigma^{(\mathcal{M})}$ \\
\hline $\begin{array}{l}\varepsilon_{l} \text { of the } w \text {-th LARS iteration is more than twice } \\
\text { that of the }(w-1) \text { th LARS iteration. }\end{array}$ & $\begin{array}{l}\text { a) Terminate LARS procedure. } \\
\text { b) Calculate } \sigma^{(\mathcal{M})} \text { in }(13) \text { using the elements in } \mathcal{Q} \text { obtained at the }(w-1) \text { th LARS iteration. }\end{array}$ \\
\hline$\varepsilon_{l} \leq \varepsilon_{t}$ before $\frac{\mathcal{L}}{4}$ LARS iterations. & $\begin{array}{l}\text { a) Set } \varepsilon_{t} \text { to half of its originally set } \varepsilon_{t} \text {. } \\
\text { b) Repeat Step 3a until at least } \frac{\mathcal{L}}{4} \text { polynomial bases are included in } \mathcal{Q} \text {. } \\
\text { c) Calculate } \sigma^{(\mathcal{M})} \text { in (13) using the elements in } \mathcal{Q} \text {. }\end{array}$ \\
\hline $\begin{array}{l}\varepsilon_{l} \leq \varepsilon_{t} \text { and the number of LARS iterations is larger } \\
\text { than } \frac{\mathcal{L}}{4} \text { and less than } \frac{5 \mathcal{L}}{6} .\end{array}$ & $\begin{array}{l}\text { a) Calculate } \hat{\sigma}^{2} \text { of the } 15|\boldsymbol{E}|^{2} \text { in (12). } \\
\text { b) Let the LARS procedure execute further } \frac{\mathcal{L}}{6} \text { LARS iterations and calculate }\left(\sigma^{(\mathcal{M})}\right)^{2} \text { in }(13) \text { whose } \\
\text { corresponding } \varepsilon_{l} \text { is less than } \varepsilon_{t} \text { at each LARS iteration. } \\
\text { c) Compare } \hat{\sigma}^{2} \text { with the variance calculated at each LARS iteration and choose the closest }\left(\sigma^{(\mathcal{M})}\right)^{2} \\
\text { to the sample variance as the final result. }\end{array}$ \\
\hline $\begin{array}{l}\varepsilon_{l} \leq \varepsilon_{t} \text { and the number of LARS iterations is larger } \\
\text { than } \frac{5 \mathcal{L}}{6} \text { or equal to } \frac{5 \mathcal{L}}{6} \text {. } \\
\varepsilon_{l}>\varepsilon_{t} \text { and the number of LARS iterations equals } \\
\mathcal{L} \text {. }\end{array}$ & Calculate $\sigma^{(\mathcal{M})}$ in (13) using the elements incorporated in $\mathcal{Q}$. \\
\hline
\end{tabular}

THE POSSIBLE CONDITIONS IN THE LARS PROCEDURE AND THE CORRESPONDING STEPS TO CALCULATE $\sigma^{(\mathcal{M})}$

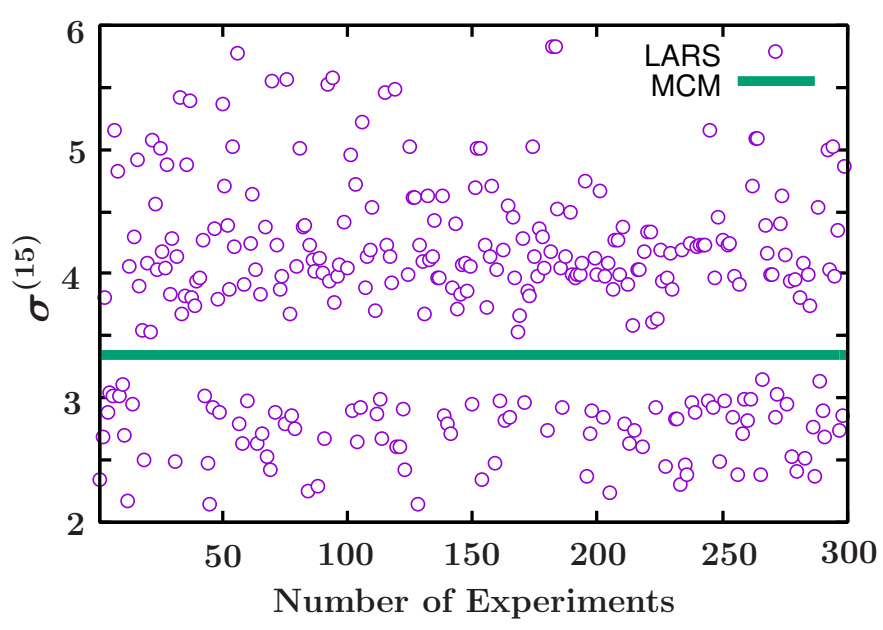

(a) $300 \sigma^{(15)}$ from Fig. 9 more than $\pm 5 \%$ of the $\sigma\left(10^{4}\right)$ of MCM.

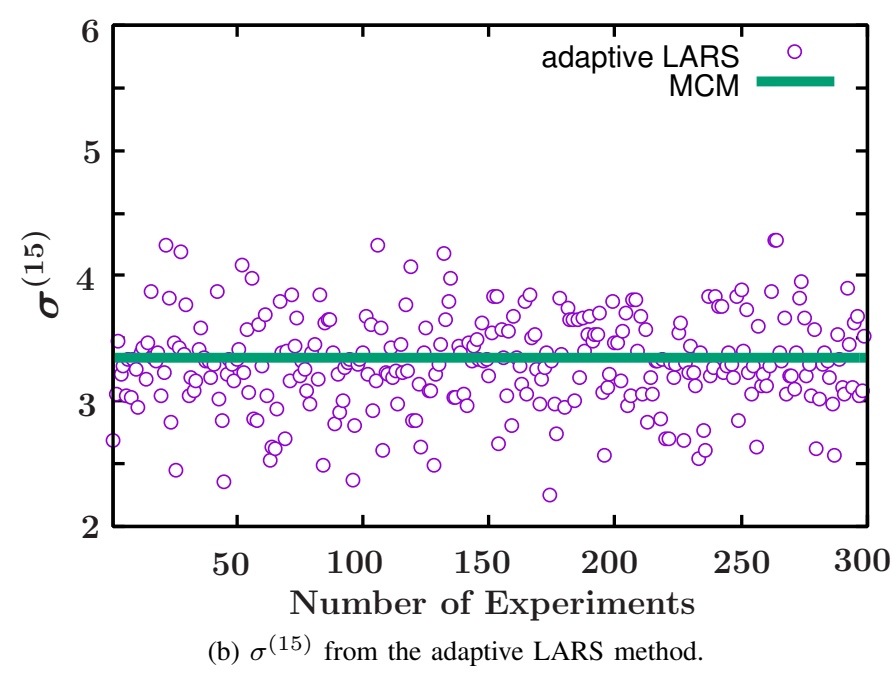

Fig. 10. (a) Unsatisfactory $\sigma^{(15)}$ obtained from the LARS method. (b) the corresponding $\sigma^{(15)}$ from the adaptive LARS method.

\section{B. Discussions}

$300 \sigma^{(15)}$ out of $1000 \sigma^{(15)}$ in Fig. 9 are improved by the adaptive LARS method as shown in Fig. 10.

The numerical experiments for UQ involve 10 Debye parameters which can be expanded with 65 polynomial terms by the general full PC truncation scheme. By using the hyperbolic scheme, the number of polynomial terms is reduced from 65 to 20, and thus the required number of the FDTD simulations is reduced to 20 as well. By determination of the optimal value of $\mathcal{M}$ applying the $\mathrm{L} 2$ regularisation scheme, the number of the required FDTD simulations is further reduced to 15 , thereby optimally reducing the computational cost. Although the process of determining $\mathcal{M}$ makes the adaptive LARS method more complex than the conventional LARS method, the computational cost of the entire process of the adaptive LARS method is still significantly lower than the conventional LARS method due to reduction of the required FDTD simulations and we achieve higher accuracy than the original LARS method.

\section{CONCLUSIONS}

The curse of dimensionality recurs in almost all UQ techniques in circumstances which involve a high number of input parameters. To overcome this problem, this paper introduced the hyperbolic scheme in the NIPC expansion to create a sparse NIPC expansion. The L2 regularisation scheme was applied to the LARS method to enable the matrix inversion to be performed irrespective of the number of samples. This allowed the LARS method to be successfully completed using only 15 FDTD simulations for 10 input variables, whereas the required number of FDTD simulations would be at least 65 in the conventional LARS method. As a consequence, the computational cost was only $23.1 \%$ of the general NIPC expansion. The proposed adaptive LARS method mainly focuses on the optimisation of the LARS method with respect to the accuracy. Its contribution lies in the analysis of the termination criterion of the LARS procedure. However, it also shows a better stability than the LARS method since it reduces the impact of the inadequate samples. 


\section{ACKNOWLEDGEMENT}

This work was completed in part with HOKUSAIGreatWave Computer System at RIKEN. http://i.riken.jp/ download/sites/2/HOKUSAI_system_overview_en.pdf.

\section{REFERENCES}

[1] J. C. Liberti and T. S. Rappaport, Smart antennas for wireless communications: IS-95 and third generation CDMA applications. Prentice Hall PTR, 1999.

[2] K. Sairam, N. Gunasekaran, and S. R. Redd, "Bluetooth in wireless communication," IEEE Commun. Mag., vol. 40, no. 6, pp. 90-96, 2002.

[3] S. Rathod, "Investigation of electromagnetic radiations in mumbai," IETE Journal of Education, vol. 55, no. 2, pp. 81-89, 2014.

[4] M. A. Esmekaya, C. Ozer, and N. Seyhan, "900 MHz pulse-modulated radiofrequency radiation induces oxidative stress on heart, lung, testis and liver tissues," Gen Physiol. Biophys., vol. 30, no. 1, pp. 84-89, 2011.

[5] J. C. Lin, "The blood-brain barrier, cancer, cell phones, and microwave radiation," IEEE Antennas Propag. Mag., vol. 43, no. 3, pp. 141-143, 2001.

[6] J. Wiart, A. Hadjem, M. Wong, and I. Bloch, "Analysis of rf exposure in the head tissues of children and adults," Physics in Medicine \& Biology, vol. 53, no. 13, p. 3681, 2008.

[7] M.-C. Gosselin, A. Christ, S. Kühn, and N. Kuster, "Dependence of the occupational exposure to mobile phone base stations on the properties of the antenna and the human body," IEEE Trans. Electromagn. Compat., vol. 51, no. 2, pp. 227-235, 2009.

[8] A. Taflove and S. C. Hagness, Computational electrodynamics: the finitedifference time-domain method. Artech house, 2005

[9] K. Yee, "Numerical solution of initial boundary value problems involving maxwell's equations in isotropic media," IEEE Trans. Antennas Propag., vol. 14, no. 3, pp. 302-307, 1966.

[10] P. Kosmas and C. M. Rappaport, "Time reversal with the fdtd method for microwave breast cancer detection," IEEE Trans. Microw. Theory and Tech., vol. 53, no. 7, pp. 2317-2323, 2005.

[11] K. Tekbas, F. Costen, R. Himeno, and J.-P. Berenger, "Subcell modelling of frequency dependent thin layers in the fdtd method," IEEE Trans. Antennas Propag., vol. 65, no. 4, pp. 1321-1329, 2017.

[12] N. R. Mehrabadi, R. Burgos, C. Roy, and D. Boroyevich, "Power electronics modeling and design: Using parametric and model-form uncertainty quantification to assess predictive accuracy of power converter models," IEEE Power Electron. Mag., vol. 4, no. 4, pp. 44-52, 2017.

[13] A. Arduino, M. Chiampi, F. Pennecchi, L. Zilberti, and O. Bottauscio, "Monte carlo method for uncertainty propagation in magnetic resonancebased electric properties tomography," IEEE Trans. Magn., vol. 53, no. 11, pp. 1-4, 2017.

[14] C. Yardim, P. Gerstoft, and W. S. Hodgkiss, "Estimation of radio refractivity from radar clutter using bayesian monte carlo analysis," IEEE Trans. Antennas Propag., vol. 54, no. 4, pp. 1318-1327, 2006.

[15] Y. Chen, J. Wen, and S. Cheng, "Probabilistic load flow method based on nataf transformation and latin hypercube sampling," IEEE Trans. Sustainable Enery., vol. 4, no. 2, pp. 294-301, 2013.

[16] R. B. Thakur, Q. Y. Tang, R. McGeehan, F. Carter, and E. Shirokoff, "Characterizing quality factor of niobium resonators using a markov chain monte carlo approach," IEEE Trans. Appl. Supercond., vol. 27, no. 4, pp. 1-5, 2017.

[17] H. Acikgoz and R. Mittra, "Stochastic polynomial chaos expansion analysis of a split-ring resonator at terahertz frequencies," IEEE Trans. Antennas Propag., vol. 66, no. 4, pp. 2131-2134, 2018.

[18] X. Cheng and V. Monebhurrun, "Application of different methods to quantify uncertainty in specific absorption rate calculation using a cadbased mobile phone model," IEEE Trans. Electromagn. Compat., vol. 59, no. 1, pp. 14-23, 2017.

[19] K. Li, T.-Z. Huang, L. Li, S. Lanteri, L. Xu, and B. Li, "A reducedorder discontinuous galerkin method based on pod for electromagnetic simulation," IEEE Trans. Antennas Propag., vol. 66, no. 1, pp. 242-254, 2018.

[20] I. Jeong, B.-G. Gu, J. Kim, K. Nam, and Y. Kim, "Inductance estimation of electrically excited synchronous motor via polynomial approximations by least square method," IEEE Trans. Ind. Appl., vol. 51, no. 2, pp. 1526-1537, 2015.

[21] G. Blatman and B. Sudret, "Adaptive sparse polynomial chaos expansion based on least angle regression," Journal of Computational Physics, vol. 230, no. 6 , pp. 2345-2367, 2011.
[22] G. E. Box, J. S. Hunter, and W. G. Hunter, Statistics for experimenters: design, innovation, and discovery, vol. 2. Wiley-Interscience New York, 2005.

[23] B. Efron, T. Hastie, I. Johnstone, and R. Tibshirani, "Least angle regression," The Annals of statistics, vol. 32, no. 2, pp. 407-499, 2004

[24] P.-Y. Wu, C.-C. Fang, J. M. Chang, and S.-Y. Kung, "Cost-effective kernel ridge regression implementation for keystroke-based active authentication system," IEEE Trans. Cybern., vol. 47, no. 11, pp. 39163927, 2017.

[25] C. Soize and R. Ghanem, "Physical systems with random uncertainties: chaos representations with arbitrary probability measure," SIAM Journal on Scientific Computing, vol. 26, no. 2, pp. 395-410, 2004.

[26] A. M. Molinaro, R. Simon, and R. M. Pfeiffer, "Prediction error estimation: a comparison of resampling methods," Bioinformatics, vol. 21, no. 15 , pp. 3301-3307, 2005.

[27] L. Lian, A. Liu, and V. K. Lau, "Weighted lasso for sparse recovery with statistical prior support information," IEEE Trans. Signal Process., 2018.

[28] J. F. Kenney, Mathematics of statistics. D. Van Nostrand Company Inc; Toronto; Princeton; New Jersey; London; New York,; Affiliated EastWest Press Pvt-Ltd; New Delhi, 2013.

[29] J.-P. Berenger, "A perfectly matched layer for the absorption of electromagnetic waves," Journal of computational physics, vol. 114, no. 2, pp. 185-200, 1994

[30] S. D. Gedney, G. Liu, J. A. Roden, and A. Zhu, "Perfectly matched layer media with cfs for an unconditionally stable adi-fdtd method," IEEE Trans. Antennas Propag., vol. 49, no. 11, pp. 1554-1559, 2001.

[31] "The RIKEN webpage, media parameters for the Debye relaxation model," http://cfd-duo.riken.jp/cbms-mp/. Accessed: Oct. 3, 2017.

\section{APPENDIX A}

MAThematical NOTATIONS

$\psi \quad$ Polynomial basis

$r \quad$ Order of NIPC expansion

$\mathcal{K} \quad$ Number of Debye parameters of interest

$\xi \quad$ Debye parameters of interest

$\alpha \quad$ Index of polynomial basis

$\boldsymbol{\alpha} \quad$ Collective form of $\alpha_{1} \alpha_{2} \cdots \alpha_{\mathcal{K}}$

$\mathcal{L} \quad$ Number of polynomial bases in the NIPC expansion

$\mathcal{M} \quad$ Number of samples

$\mathcal{T}\left(\boldsymbol{\xi}^{(m)}\right) \quad$ Predicted value of $|\boldsymbol{E}|^{2}$ for $\boldsymbol{\xi}^{(m)}$

$\widehat{\mathcal{T}}\left(\boldsymbol{\xi}^{(m)}\right) \quad$ Current LARS update of $\mathcal{T}\left(\boldsymbol{\xi}^{(m)}\right)$

a Coefficient of $\psi$

$\mathcal{C}$ Correlation between $\psi$ and the residual vector

$\mathcal{E} \quad$ Vector of $\mathcal{M}|\boldsymbol{E}|^{2}$

$\hat{\Psi} \quad$ Matrix of the chosen $\psi$

$\hat{\boldsymbol{a}} \quad$ Vector of the coefficients of the chosen $\psi$

$\widetilde{k} \quad$ Index of the most correlated polynomial basis

$\mathcal{Q} \quad$ A set containing the chosen $a_{\boldsymbol{\alpha}_{\tilde{k}}} \psi_{\boldsymbol{\alpha}_{\tilde{k}}}$

$\hat{\gamma} \quad$ Update coefficient of the LARS method

$w \quad$ Index of LARS iteration

$\varepsilon_{t} \quad$ Target error

$\varepsilon_{l} \quad$ LOO error

$\varrho \quad$ Regularisation factor

$\zeta \quad$ Minimum number of polynomial bases in $\mathcal{Q}$

$\iota \quad$ Number of additional LARS iterations

$\varsigma \quad$ Intermediate upperlimit of the number of LARS iterations

$\nu(\mathcal{M}) \quad$ Average value from the MCM

$\sigma(\mathcal{M}) \quad$ Standard deviation from the MCM

$\sigma^{(\mathcal{M})}$ Standard deviation from the LARS and adaptive LARS methods with $\mathcal{M}$ samples 